\title{
Avaliação da quantidade de óleo de soja em refeições oferecidas em um restaurante universitário
}

\author{
Assessing the amount of soybean oil in meals offered in a university restaurant
}

Cláudia Fernanda dos Santos Calixto Joyce Fernandes Menezes Azevedo' Marlene Azevedo Magalhães Monteiro² Marco Antônio Schaefer ${ }^{3}$

\footnotetext{
1 Discente do Curso de Nutrição da Universidade Federal de Minas Gerais - UFMG. E-mail: claudiafsc@yahoo.com.br; ioyce fernandes2005@yahoo.com.br ${ }^{2}$ Docente do Curso de Nutrição, Departamento de Enfermagem Aplicada, Universidade Federal de Minas Gerais - UFMG.E-mail:marleneaz@ enf.ufmg.br

${ }^{3}$ Nutricionista do Restaurante Universitário da Universidade Federal de Minas Gerais, Fundação Mendes Pimentel - FUMP. E-mail: setorial@ fump.ufmg.br

Correspondência / Correspondence Marlene A. Magalhães Monteiro Universidade Federal de Minas Gerais-UFMG Escola de Enfermagem Av. Prof. Alfredo Balena, 190 - $5^{\circ}$ andar - Sala 518 - Bairro Santa Efigênia 30130-100 - Belo Horizonte, Minas Gerais, Brasil E-mail:marleneaz@enf.ufmg.br
}

\section{Resumo}

Este estudo teve como objetivo avaliar a quantidade de óleo de soja oferecida em um restaurante universitário do município de Belo Horizonte-MG, e compará-la com o preconizado pela Organização Mundial da Saúde (OMS). A coleta de dados foi iniciada após capacitação dos funcionários do local, visando à separação de óleos provindos das fritadeiras e da gordura desprendida dos alimentos, com armazenamento dos mesmos em locais diferentes (carro para transporte de alimentos e tambor, respectivamente). Mensurou-se o óleo de soja rejeitado proveniente das fritadeiras e fornecido para cocção dos alimentos, além do número de comensais. Os dados foram obtidos pelo controle realizado com auxílio do software da Teknisa ${ }^{\circledR}$. O valor per capita encontrado para a oferta de óleo de soja por refeição, almoço e jantar, considerando o volume de rejeito, foi de $15,5 \mathrm{~mL}$ e $8,6 \mathrm{~mL}$, respectivamente, e o valor médio total de $14,96 \mathrm{~mL}$ abaixo do preconizado pela OMS (16 mL/dia). Concluiu-se que a média de oferta do óleo de soja per capita está abaixo de valores considerados adequados para um dia. Entretanto, o valor encontrado corresponde ao oferecido em apenas uma refeição (almoço), o que poderá implicar extrapolação da ingestão diária desse alimento recomendada por um dia, devendo o serviço rever seus procedimentos de produção de refeições.

Palavras-chave: Serviços de alimentação. Óleo de soja. Saúde. 


\section{Abstract}

This study aimed to evaluate the amount of soybean oil offered in a university restaurant in the city of Belo Horizonte-MG, and compare it with World Health Organization (WHO) recommendations. Data collection was initiated after training the staff, aimed at separating oil fryers from the detached fat foods, with storage facilities in different locations (car for transporting food and barrel, respectively). We measured soybean oil rejected from fryers and used for cooking food, and the number of diners. Data were obtained for the control obtained with through the software Teknisa ${ }^{\circledR}$. The per capita value found for soybean oil supply by meal, lunch and dinner, considering the volume of tailings was $15.5 \mathrm{~mL}$ and $8.6 \mathrm{~mL}$, respectively, and the average total of $14.96 \mathrm{~mL}$ was lower than recommended by WHO (16 $\mathrm{mL} /$ day). It was concluded that the average supply of soybean oil per capita is below values considered adequate for one day. However, this value corresponds to that offered in just one meal (lunch), which may imply extrapolating the recommended daily intake of that food for a day, and the service should review its procedures for meals production.

Key words: Food services. Soybean oil. Health.

\section{Introdução}

No Brasil, nos últimos 35 anos verificou-se diminuição no consumo de alimentos tradicionais, como arroz e feijão, simultaneamente ao aumento no consumo de óleos vegetais, alimentos de origem animal, como carnes, embutidos e leite e derivados, refrigerantes e biscoitos, além de permanência do consumo excessivo de açúcar e insuficiente em frutas e hortaliças, características que revelam um padrão alimentar inadequado, com dietas pobres em alguns micronutrientes e fibras e alta densidade energética. ${ }^{1,2}$

Na nutrição humana, os óleos têm importância significativa, uma vez que são ricos em ácidos graxos insaturados (ácido oleico, ácido linoleico e alfa-linoleico) e, pobres em ácidos graxos saturados, além de serem veiculadores de vitaminas lipossolúveis e fornecedores de energia. ${ }^{3-6}$ Em que pesem tais qualidades nutricionais, a ingestão excessiva de óleos vegetais vem se dando, em particular, por meio de processos de fritura ${ }^{6}$, cuja operação de preparação rápida confere aos alimentos características únicas de saciedade, aroma, sabor e palatabilidade. ${ }^{7}$

Durante a fritura, ocorrem mudanças físicas no óleo, dentre as quais o escurecimento, o aumento da viscosidade, a diminuição do ponto de fumaça e a formação de espuma. 
Quanto às alterações químicas, há a hidrólise para a formação de ácidos graxos livres, monoacilglicerol e diacilglicerol; a oxidação, resultando em peróxidos, hidroperóxidos, dienos conjugados, epóxidos, hidróxidos e cetonas; e a decomposição em pequenos fragmentos. ${ }^{8}$

Além disso, óleos e gorduras, sob condição de fritura, são levados à formação de isômeros geométricos trans dos ácidos graxos oleico, linoleico e $\alpha$-linolênico. ${ }^{9}$ Evidências indicam que uma pequena ingestão de ácidos graxos trans pode afetar o perfil de lipoproteínas, diminuindo a lipoproteína de alta densidade (HDL) e aumentando a lipoproteína de baixa densidade (LDL) e lipoproteína a (Lpa). ${ }^{10}$ Desta forma, o consumo elevado de ácidos graxos trans pode ser importante fator de risco para as doenças do coração. ${ }^{11}$

Com base em estudos epidemiológicos recentes, a Organização Mundial da Saúde (OMS) recomenda que o consumo máximo deste tipo de gordura não deve ser superior a $1 \%$ das calorias totais. ${ }^{6}$ Assim, o nutricionista, como profissional da área da saúde e no exercício de suas atribuições em unidades de alimentação e nutrição, além de planejar, organizar, dirigir, supervisionar e avaliar os serviços de alimentação e nutrição, deve realizar assistência e educação nutricional a coletividade ou indivíduos sadios ou enfermos em instituições públicas e privadas. ${ }^{12}$

Considerando tais atribuições, a mudança no perfil nutricional da população e o crescente aumento das DANTs, o objetivo deste trabalho foi avaliar a quantidade de óleo vegetal oferecida em um restaurante universitário e relacioná-la ao preconizado pela OMS.

\section{Material e métodos}

O restaurante universitário, local onde foi realizado este estudo, situa-se no município de Belo Horizonte-MG e funciona através do sistema de subsídio financeiro governamental, com preços diferenciados segundo a categoria socioeconômica (Nível I: estudantes que apresentam grande dificuldade para se manter na universidade; Nível II: estudantes que apresentam nível de dificuldade intermediária para se manter na universidade; e Nível III: estudantes que apresentam baixo grau de dificuldade para se manter na universidade), conforme Portaria n. 004/2005 da UFMG, ${ }^{13}$ a qual exige vinculação do usuário à universidade ou visitante.

O restaurante serve uma média acima de 6.000 refeições por dia, incluindo desjejum (300 refeições), almoço (5.000 refeições) e jantar (700 refeições). O horário de funcionamento é de segunda à sexta-feira (exceto feriados), das $6 \mathrm{~h} 45 \mathrm{~min}$ às $8 \mathrm{~h} 10 \mathrm{~min}$, das $11 \mathrm{~h}$ às $14 \mathrm{~h}$ e das $17 \mathrm{~h} 30 \mathrm{~min}$ às 19h. E aos sábados (exceto feriados), das 11h30min às 13h. Os cardápios dos desjejuns são constituídos por pãozinho de sal com margarina, café, achocolatado e leite, e uma fruta; e as 
grandes refeições, por prato principal (três opções), guarnição, salada, sobremesa, arroz, feijão e refresco. O sistema de distribuição de refeições adotado é o self-service, exceto para o prato principal e guarnição, que são porcionados por um funcionário do restaurante. O óleo utilizado no preparo das refeições é o de soja.

Os usuários atendidos são estudantes, servidores da instituição de ensino e de órgãos e fundações de apoio da universidade. No período avaliado, as refeições almoço e jantar foram servidas durante 22 e 19 dias, respectivamente, um total de 99.405 refeições, sendo 91.240 no almoço e 8.165 no jantar.

Sob supervisão dos pesquisadores, foi desenvolvida pelos estudantes do curso de Nutrição uma capacitação participativa e dialógica por meio de atividades lúdicas para os funcionários do restaurante, enfatizando a relação entre fundamentação teórica e prática sobre o tema deste estudo e dos procedimentos para a coleta do material.

A capacitação foi realizada antes da coleta de dados, com duração de 15 minutos, tendo como público-alvo sete cozinheiros e cinco auxiliares de cozinha. Nela foram abordados o objetivo do projeto; a diferença entre a gordura presente no alimento e a que é acrescentada; a relação entre óleos e gorduras; a atuação do nutricionista e a saúde; e principalmente, a importância da separação de óleo vegetal proveniente das fritadeiras e a gordura proveniente dos alimentos para a realização deste estudo.

Na coleta dos dados do óleo de soja, foram utilizados um carrinho basculante para transporte de alimentos (450 x 675 x 750 milímetros) para armazenar óleo proveniente exclusivamente das fritadeiras; um tambor (200 litros) para armazenar demais gorduras advindas do outros processos de preparo dos alimentos (frigideiras e fornos); uma régua de acrílico de 50 centímetros e um formulário próprio (dia, tipo de óleo coletado, quantidade) para registro dos dados. A coleta e análise dos dados basearam-se em uma adaptação da determinação de volume por deslocamento de água. ${ }^{14}$

No período de coleta de dados, entre 30 de outubro a 30 de novembro de 2010, foram realizadas duas medições, adotando os mesmos princípios e recipiente. As medições foram realizadas de acordo com a necessidade de reposição do óleo de soja nas fritadeiras, sem interferência direta dos cardápios preparados nesse período.

Os dados coletados são referentes exclusivamente ao óleo de soja proveniente das fritadeiras. Demais gorduras originárias do alimento foram descartadas, por não serem óleo de adição. A quantidade de óleo vegetal fornecida para a cocção dos alimentos e o número de comensais no período (almoço e jantar) foram obtidos a partir do controle realizado com o auxílio do software Tecfood, da Teknisa ${ }^{\circledR}$, versão 4.09.069. 
Os dados ao serem convertidos em litros forneceram o volume de óleo desprezado. A partir desses dados, estabeleceu-se que para cada $18 \mathrm{~L}$ de óleo havia uma equivalência média de 9,25 cm, e para cada $1 \mathrm{~L}$ de óleo, o valor seria de $0,51 \mathrm{~cm}$. Por meio do cálculo por diferença, foi possível encontrar o teor de óleo de soja utilizado nas preparações do almoço e jantar juntos. Não houve utilização de óleo de soja no preparo do desjejum. Tal resultado permitiu inferir a quantidade de óleo de soja utilizada na produção das preparações per capita. Os resultados obtidos foram comparados com o preconizado pela OMS.

\section{Resultados}

Durante a realização do estudo, foram disponibilizados pelo setor de estocagem para o preparo dos alimentos (almoço e jantar), 1.553,7 litros de óleo. Para o preparo dos alimentos servidos no almoço, foram utilizados 1.479,9 litros, o que corresponde a aproximadamente $95 \%$ do total (figura 1).

\section{Quantidade de óleo (L)}

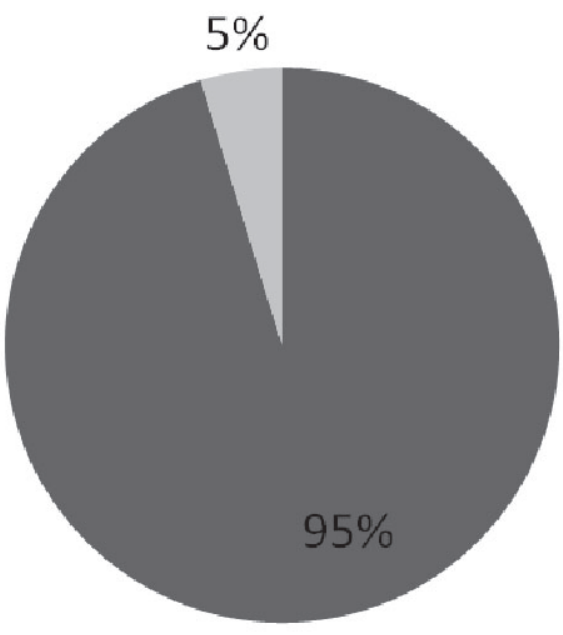

Almoço

$1479,9 \mathrm{~L}$

Jantar

$73,8 \mathrm{~L}$

\section{Total: $1553,7 \mathrm{~L}$}

Figura 1. Quantidade de óleo utilizada por refeição em um restaurante universitário, Belo Horizonte-MG, 2010. 
Nesse período, o volume total de óleo de soja descartado das fritadeiras foi de 66,27 litros (figura 2). Desta forma, pode-se estimar por diferença que o consumo de óleo foi de 1.487,43 litros, com um per capita de 14,96 mL/refeição, para almoço e jantar. Considerando a proporção de óleo utilizada somente para o almoço (95\%) e para o jantar (5\%), e mantendo a mesma proporção para o volume de óleo descartado, o per capita passa a ser de 15,5mL para o almoço e $8,6 \mathrm{~mL}$ para o jantar (figura 2).

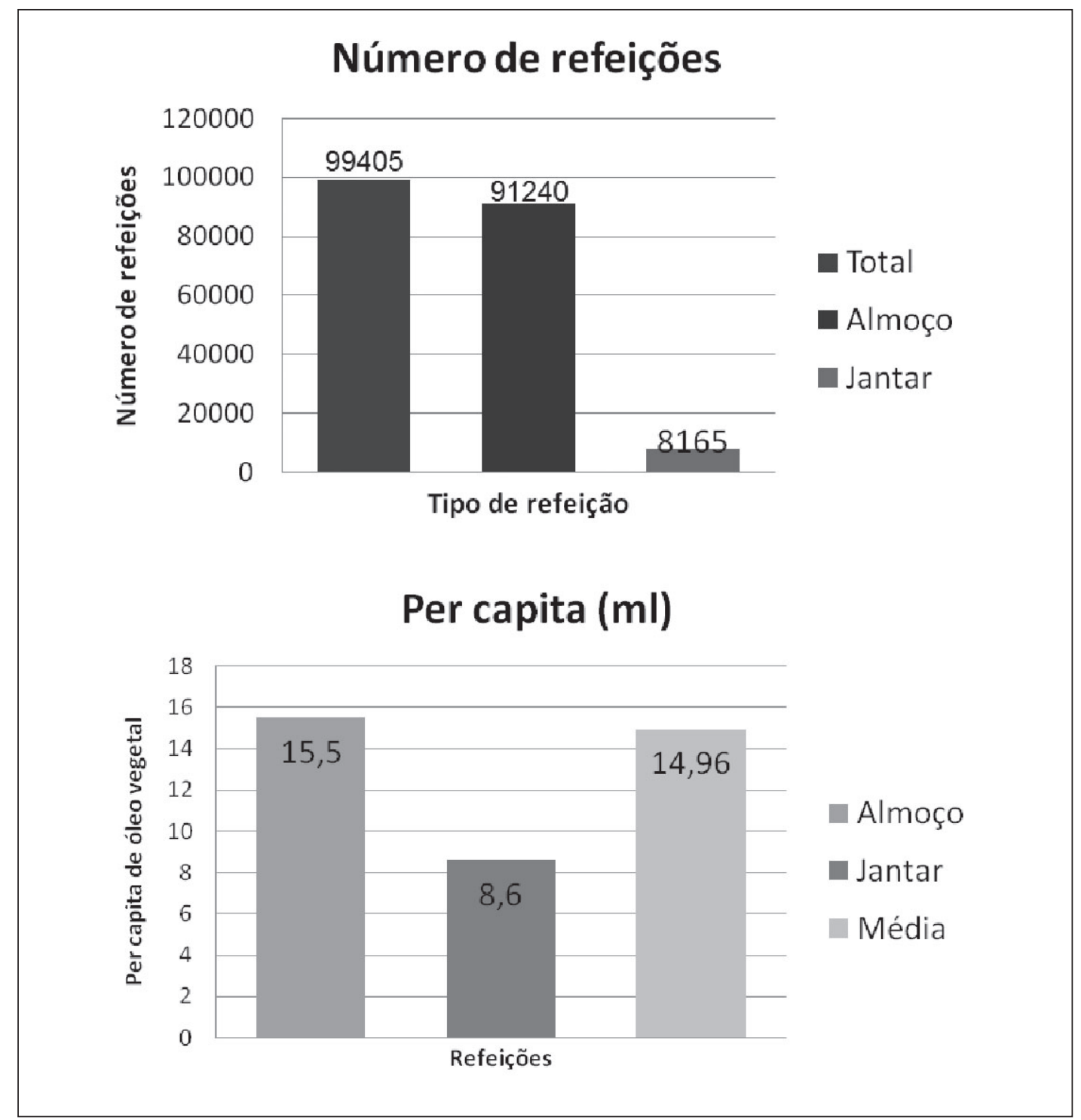

Figura 2. Quantidade de óleo de soja per capita segundo refeições oferecidas em um restaurante universitário, Belo Horizonte-MG, 2010. 


\section{Discussão}

O número de refeições servidas diariamente pelo restaurante universitário em geral é superior a 6.000 refeições; no entanto, a média mensal desse período foi de 4.730 refeições, em virtude do término do período letivo e por ser um período chuvoso. Por este motivo, os resultados obtidos não podem ser replicados para os demais períodos do ano.

Pode-se afirmar que a quantidade de óleo de soja oferecida, considerando a média das duas refeições (almoço e jantar), foi estimada em 14,96 mL per capita. De acordo com a OMS, ${ }^{15}$ a ingestão de óleos e gorduras não deve ultrapassar duas porções diárias, o equivalente a $16 \mathrm{~mL} /$ pessoa (uma colher de sopa cheia). Desta forma, o valor per capita de óleo de soja está próximo ao recomendado, sem considerar a ingestão dos outros alimentos e fontes de gordura ao longo do dia.

Além disso, considerando que, em média, 94\% dos indivíduos afirmam realizar ao menos uma grande refeição/dia (almoço ou jantar), ${ }^{16}$ e que o per capita de óleo de soja para o almoço neste estudo é de 15,5mL, reforça-se a tendência de uma ingestão diária acima $16 \mathrm{~mL} /$ pessoa.

Amorim, Junqueira \& Jokl ${ }^{17}$ avaliaram que as quantidades médias de gorduras per capita oferecidas no almoço foram de 21,6mL, incluindo margarina, maionese e azeite. E em uma unidade de alimentação hospitalar, Almeida et al. ${ }^{18}$ apuraram o total de 18, 8土 3,2mL de óleo por refeição produzida, baseados na diferença entre a quantidade média mensal de óleo requisitada ao almoxarife para a produção das refeições e a quantidade média mensal de óleo descartada para reciclagem. Em ambos os estudos, os valores encontrados também foram superiores ao recomendado pela OMS.

Visando reduzir o consumo de óleo de soja, o restaurante universitário tem utilizado métodos de cocção alternativos, como o de ar quente, por meio de fornos convencionais e combinados, evitando preparações à base de frituras. Esse fato foi observado durante o período deste estudo. Araújo $^{19}$ afirma que a utilização de gorduras em alguns métodos é imprescindível, porém na maioria, o uso pode ser reduzido ou eliminado, utilizando apenas ar quente.

Ao analisar o cardápio ofertado pela unidade de alimentação, além do óleo de soja utilizamse rotineiramente outras fontes de gorduras, como margarina, maionese e azeite de oliva, que não foram avaliados neste estudo, mas estão incluídos na recomendação da OMS. Assim, o valor médio per capita obtido neste estudo está subdimensionado em relação à real quantidade de óleo de soja oferecida pelo restaurante a seus usuários.

A padronização das fichas técnicas é uma ferramenta a ser implantada para garantir a qualidade nutricional das refeições, pois ajuda a controlar o valor energético oferecido, sobretudo com relação ao óleo vegetal. ${ }^{17}$ No restaurante universitário, apesar da supervisão contínua por parte 
do nutricionista e da técnica em nutrição durante a preparação das refeições, os cozinheiros não replicam as fichas técnicas em sua totalidade.

Todo o óleo rejeitado decorrente do processo de fritura por imersão, bem como a gordura desprendida das preparações assadas são coletados por uma empresa com autorização ambiental. A utilização de resíduos gordurosos, por parte dessa empresa, destina-se à produção para obtenção de biodiesel, massa para vidros, sabão e demais produtos biodegradáveis.

\section{Conclusão}

Este estudo demonstrou que apesar de a média de oferta do óleo de soja do restaurante universitário estar abaixo do preconizado pela OMS, tal valor pode não refletir a ingestão real do seu usuário, em virtude das demais refeições realizadas ao longo do dia e consumo de outras fontes de gorduras.

Assim, diante à mudança no perfil nutricional da população e o crescente aumento das doenças e agravos não-transmissíveis, faz-se necessário analisar e adequar, permanentemente, a oferta de óleos e gorduras nas preparações servidas neste restaurante.

\section{Referências}

1. Levy-Costa RB, Sichieri R, Pontes NS, Monteiro CA. Disponibilidade domiciliar de alimentos no Brasil: distribuição e evolução (1974-2003). Rev Saúde Pública 2005; 39:530-40.

2. IBGE. Pesquisa de Orçamentos Familiares 2008/2009: avaliação nutricional da disponibilidade domiciliar de alimentos no Brasil. Rio de Janeiro: IBGE; 2010.

3. Kratz M. Dietary mono and polyunsaturated fat acids similarly affect LDL size in healthy men and women. J Nutr. 2002; 132(04): 715-8.

4. Ornellas L. Técnica dietética: seleção e preparo de alimentos. 7. ed., São Paulo: Atheneu; 2001.

5. Gregório BM, Andrade ECB. Influência do aquecimento sobre as propriedades físico-químicas de óleos comestíveis. Hig aliment. 2004; 18(124): 78-84.

6. Cella RCF, Regitano-D’arce MAB, Spoto MHF. Comportamento do óleo de soja refinado utilizado em fritura por imersão com alimentos de origem vegetal. C Tecn Alim. 2002; 22(2): 111-6.

7. Agência Nacional de Vigilância Sanitária (Brasil). Informe técnico no 11. [Acesso em 15 out. 2012]. Disponível em:http://www.anvisa.gov.br/alimentos/informes/11_051004.htm

8. Sanibal EAA, Mancini-Filho J. Perfil de ácidos graxos trans de óleo e gordura hidrogenada de soja no processo de fritura. Cienc tecnol aliment. 2004; 24(1): 27-31. 
9. Bretillon L, Chardigny JM, Noel JP, Sebedio JL. Desaturation and chain elongation of [1-14C] monotrans isomers of linoleic and a-linolenic acids in perfused rat liver. J Lipid Res. 1998; 39: 2228-36.

10. Ascherio A, Willett WC. Health effects of trans fatty acids. Am J Clin Nutr. 1997; 66(4):1006-10.

11. Ferreira SRG. Alimentação, nutrição e saúde: avanços e conflitos da modernidade. Cienc Cult. 2010; 62(4): 31-3.

12. Conselho Federal de Nutrição (Brasil). Resolução 380/2005. Atribuições do Nutricionista. [Acesso em 15 out. 2010]. Disponível em: http://www.cfn.org.br/novosite/pdf/res/2005/res380.pdf.

13. Universidade Federal de Minas Gerais. Portaria 004/2005 de 13 de janeiro de 2005: Estabelecer os seguintes valores a serem pagos, por refeição. Belo Horizonte, 13 de janeiro de 2005. [Acesso em 11 nov. 2011]. Disponível em: https://www.ufmg.br/online/arquivos/2005/01/restaurantes_ universitrios_ter.shtml.

14. Ferreira SMR. Controle de qualidade em sistemas de alimentação coletiva. São Paulo: Livraria Varela, 2002.

15. World Health Organization. Obesity: preventing and managing the global epidemic. Report of a WHO consultation on obesity. Geneva: World Wealth Organization; 2000.

16. Barbosa L. Feijão com arroz e arroz com feijão: o Brasil no prato dos brasileiros. Horiz Antrop. 2007; 28: 87-116.

17. Amorim MMA, Junqueira RG, Jokl L. Consumo de óleo e gordura nas preparações do almoço self service. Alim e Nutr. 2010; 21(2): 217-23.

18. Almeida JMA, Jardim-Botelho A, Amorim MMA. Quantidade de óleo empregada no preparo das dietas hospitalares. In: Congresso Brasileiro de Ciência e Tecnologia de Alimentos, 21, 2008, Belo Horizonte. Resumos. Belo Horizonte:SBCTA/MG, 2008. CD-ROM.

19. Araújo VMC. Alquimia dos alimentos. 2. ed. Brasília: Senac; 2008.

Recebido: $18 / 10 / 2012$

Revisado: 25/1/2013

Aprovado: $18 / 2 / 2013$ 
